\title{
Gentherapeutische Ansätze in der gynäkologischen Onkologie
}

\author{
T. Bauknecht \\ Universitäts-Frauenklinik Bonn
}

Krebs kann allgemein als eine genetische Erkrankung angesehen werden, bei der eine Abfolge verschiedener Genveränderungen aufgetreten ist. Die Kaskade dieser Genveränderungen führt schließlich zu dem malignen Phänotyp, wobei bestimmte Wächtergene (Gatekeeper) von besonderer Bedeutung sind. Damit wird ersichtlich, welche komplexen Anforderungen an ein gentherapeutisches Konzept gestellt werden. Grundsätzlich handelt es sich ja bei der Gentherapie um das Einbringen genetischer Informationen in Zellen von Lebewesen zum Zwecke der Therapie. Die Voraussetzungen der Gentherapie sind:

- Effizienter Transfer,

- konstitutive oder transiente Genexpression,

- DNA-Integration,

- möglichst hohe Tumorspezifität.

Schließlich ist auch die Therapiesicherheit ein Anliegen, was meist nur mit hohem finanziellen Aufwand (sogenannte GMP Facility) gewährleistet ist. Für den Gentransfer bedient man sich häufig der Genfähren (Vektoren), die üblicherweise viralen Ursprungs sind (Retroviren, Adenoviren, Adeno-assoziierte Viren u.a.). Auch non-viraler Gentransfer über Rezeptoren, Liposomen, Ribozyme, die Elektroporation, Gene-gun und die Injektion nackter DNA stellen weitere Ansätze dar. Ein ungelöstes Problem ist nach wie vor die Effizienz des Gentransfers. Der am häufigsten benutzte Ansatz ist der Gentransfer mit Retroviren, wobei die für die Infektion und Proliferation des Virus erforderlichen Gene (gag, env, pol) durch das therapeutische Gen ersetzt werden. Damit infektiöse Partikel entstehen, müssen sogenannte Verpackungszellen hinzugegeben werden. Ein Nachteil der Retroviren ist die geringe Titerhöhe und daß nur proliferierende Zellen infiziert werden. Ein Vorteil ist, daß das Gen durch DNA-Integration stabil transduziert wird. Adenoviren dagegen infizieren auch ruhende Zellen, wobei das Gen episomal lokalisiert bleibt und damit nur eine transiente Expression erreicht wird.

Die Ziele der Gentherapie sind:

- In-vivo-Diagnostik,

- Substitutionstherapie bei einem Gendefekt,

- Additionstherapie durch Einbringen eines Fremdgenes,

- Suppressionstherapie bei einer Überfunktion durch Onkogene. Eines der am häufigsten bearbeiteten Gentargets ist das p53, welches bei einer Vielzahl von Malignomen durch Mutation defekt ist oder auch durch Viren (z.B. HPV) inaktiviert wird. p53-Substitutionen werden durch verschiedene Technologien erzielt, wie das p53-Adenovirus, durch p53-terminale Peptide mit einer transskriptionellen Transaktivierung von Wildtyp und mutiertem p53 oder durch attenuierte Adenoviren (Onyx015), die mutierte p53 Zellen selektiv lysieren. Das p53Adenovirus wird insbesondere beim Ovarialkarzinom im Rahmen von Phase-I-Studien geprüft. Der Studienansatz versucht, beim nicht optimal operierten Ovarialkarzinom p53-Adenoviren intraperitoneal einzubringen. Mögliche Probleme des Ansatzes sind der insuffiziente Gentransfer und die widersprüchliche Datenlage der p53-Literatur als Prädiktor einer Chemotherapie. Eine weitere Möglichkeit der Gentherapie beim Zervixkarzinom ist der Nachweis varianter Formen des Matrix-Proteins CD44. In der Arbeitsgruppe um Dall (Düsseldorf) wurden Konstrukte der CD44-Varianten mit dem T-ZellRezeptor zur spezifischen Aktivierung des Immunsystems hergestellt. Andere Ansätze beim Mamma- und Ovarialkarzinom sind Her-2- und BRCA1-Konstrukte als Ansätze einer Suppressionstherapie bzw. einer Substitutionstherapie. Das Einbringen sogenannter Suizid-Gene ist eine Form einer Additionstherapie. Das Herpes-simplex-Virus Thymidin-KinaseGen (HSV-TK) wird benutzt, um eine Prodrug (Ganciclovir) umzuwandeln. Dieser Ansatz wird zur Zeit beim Ovarialkarzinom untersucht. Auf dem ASCO 99 wurde im Rahmen einer Phase-I/II-Studie der Transfer des HSV-TK mit einer nachfolgenden Kombinationsbehandlung, bestehend aus Ganciclovir und Topotecan, vorgestellt. Die Autoren waren aber nicht in der Lage, an SLO-Biopsaten den Gentransfer nachzuweisen. Die therapeutischen Ergebnisse sowie die Nebenwirkungen entsprachen wohl auch am ehesten der Wirkungsweise von Topotecan.

Zytostatika benutzen üblicherweise zur Induktion der Apoptose bestimmte Signalketten, die der Genfamilie des TNF-R zugerechnet werden können. Ein Defekt oder ein Funktionsverlust dieser Signalketten geht wahrscheinlich mit einer Resistenzentwicklung einher. Auch hier existieren schon verschiedene Ansätze wie das Einbringen von Liganden, die diese Signalwege der Caspasen regulieren, um die Resistenzentwickung zu durchbrechen.

Die Immuntherapie gewinnt zunehmende Bedeutung, vor allem in ihrer Verknüpfung mit der Gentherapie. Das zelluläre

\begin{tabular}{ll}
\hline KARGER & @ 1999 S. Karger GmbH, Freiburg \\
Fax +49 7614520714 & Accessible online at: \\
$\begin{array}{l}\text { E-mail kargergmbh@aol.com } \\
\text { www.karger.com }\end{array}$ & http://BioMedNet.com/karger
\end{tabular}

Prof. Dr. med. Thomas Bauknecht Universitäts-Frauenklinik Sigmund-Freud-Straße 25 D-53105 Bonn-Venusberg (Deutschland) Tel. 0228-2 875444, Fax -2876091 
System der Immunabwehr durch zytotoxische T-Lymphozyten ist beim Malignom häufig gestört und es wurden Ansätze entwickelt, um diesen Funktionsverlust zu rekonstituieren. Insbesondere ist die Verwendung sogenannter dendritischer Zellen (professionelle Antigen-präsentierende Zellen) interessant, die als ko-stimulatorisches Signal Zytokine zur Aktivierung zytotoxischer T-Lymphozyten freisetzen.

In der Educational Session wurde die Möglichkeit des Transfers eines mdr (Multidrug-Resistance-Gen) in hämatopoe- tische Progenitorzellen dargestellt. Präsentiert wurde die Internet-Adresse (http://www.iupui.edu/ iucc/ngvl) mit einer Übersicht über gentherapeutische Konstrukte, die zur Zeit von verschiedenen Arbeitsgruppen in den USA hergestellt und im Rahmen von klinischen Studien geprüft werden. Somit wäre es wahrscheinlich sinnvoll, sich an diesen schon laufenden klinischen Studien zu beteiligen. Für die Zukunft ist zu hoffen, daß sich in Deutschland weitere gentherapeutische Arbeitsgruppen etablieren. 